\title{
Magnetic Micro Sensor Activities at the Leibniz Universität Hannover
}

\author{
Gatzen, Hans-Heinrich, Ruffert, Christine \\ Institute for Microtechnology, Center for Production Technology, \\ Leibniz Universität Hannover, An der Universitaet 2, 30823 Garbsen, Germany \\ e-mail: ruffert@imt.uni-hannover.de, gatzen@imt.uni-hannover.de
}

\section{Introduction}

The Institute for Microtechnology (imt) at the Leibniz Universität Hannover`s Center for Production Technology (PZH) in Garbsen, Germany, is one of the world's leading institutions with a focus on the design and fabrication of magnetic micro sensors and actuators. Although there is a growing demand for the development of magnetic micro actuators for hard disk drive applications, micro positioning or fluidic control, the area of micro sensors is still dominating the market. One focus in the development of magnetic micro sensors is set on the needs of the automotive industry to optimize the driver's safety or the driving comfort.

This paper provides an overview over the activities in the area of the development and fabrication of magnetic micro sensors at the imt. The sensors cover a wide range of applications including strain, position measurement, and the detection of the magnetic properties of patternd magnetic thin-films. The sensors are either based on magneto-elastic effects for strain measurement applications or they use the inductive effect for position measurement or the characterization of magnetic thin-films used in magnetic Micro Electro-mechanical Systems (MEMS). To achieve an optimal design of the micro sensors, FEM analyses are performed using the simulation software ANSYS ${ }^{\text {TM }}$.

\section{Fabrication Technologies and Challenges}

The magnetic micro devices are fabricated in thin-film technology. For the deposition and patterning of the required hard and soft magnetic as well as the conducting and insulating material, Physical Vapor Deposition (PVD), Plasma Enhanced Chemical Vapor Deposition (PECVD) methods, and electroplating are combined with photolithography and etching processes.

In addition to the thin-film processes, Chemical-mechanical Polishing (CMP) is applied for the planarization on wafer level to provide an even surface before the next layer is deposited. The surface flatness is a precondition to achieve an optimal resolution in the following photolithography processes and consequently a high accuracy in the generated patterns. Usually, a combination of two materials such as a metal embedded in a photoresist has to be processed ${ }^{1,2}$.

Beside the availability of the respective thin-film technologies to deposit and pattern the required material, one focus has to be set on the compatibility of the applied technologies and processes. This includes chemical resistance (against e.g. etching solutions or electrolytes) as well as the thermal stability during annealing, deposition, and dry etching processes. The challenges in the fabrication of magnetic micro structures are twofold: First, the deposited structures have to feature the desired magnetic properties. For instance, for soft magnetic materials, a high saturation flux density $B_{S}$ and relative permeability $\mu_{r}$ are desirable, while hard magnetic material should feature a great coercitivity $H_{C}$ and high energy product $|B H|_{\text {max }}$. Second, adequate methods for the magnetic characterization have to be available. Furthermore, typically the magnetic thin-films are anisotropic. This requires a measurement method to detect the properties along the both magnetic axes, the easy and the hard axis of the material. A promising approach to solve this issue is presented in this paper.

\section{Characterization of Magnetic Thin-Films}

One of the main challenges in the fabrication of magnetic micro devices is to achieve the required magnetic properties in the magnetic thin-films integrated in the device. Since magnetic thin-films typically

\footnotetext{
${ }^{1}$ C. Kourouklis, T. Kohlmeier, H.H. Gatzen: The Application of Chemical-Mechanical Polishing for Planarizing a SU-8/Permalloy Combination Used in MEMS Devices. Sensors \& Actuators A: Physical, Vol. 106, No. 1-3, pp. 268-271, 2003

${ }^{2}$ H.H. Gatzen, C. Morsbach, C. Kourouklis: Chemical-mechanical Planarization of a SU-8/Copper combination for MEMS. Proc. ASPE $18^{\text {th }}$ Ann. Meet. 2003, Portland, OR, USA, pp. 575-578, 2003
} 
feature a magnetic anisotropy, it is furthermore desirable to measure the magnetic thin-film properties along the easy axis and the hard axis of the sample as well. To provide such a measurement set-up, a hysteresis sensor system was developed detecting the $B-H$ loop of anisotropic magnetic thin-films used in magnetic micro sensors on wafer level. To allow for a measurement along both the easy and the hard axis, the hysteresis sensor system consists of a pair of micro sensors arranged at an angle of $90^{\circ}$ to each other side by side on a micro actuator to be able to carry the sensors to the respective measurement location. The arrangement of the micro hysteresis sensor system is shown in Figure $1^{3}$. The footprint of a system consisting of two sensors offset by $90^{\circ}$ amounts to $1.5 \mathrm{~mm} \times 1.5 \mathrm{~mm}$.

From a functional point of view, the micro hysteresis sensors are a pair of micro transformers. The magnetic sample to be characterized forms one leg in the sensors's flux path. To achieve this set-up, the micro hysteresis sensor has a soft-magnetic core made of NiFe81/19 with a measurement gap between the upper coil and the sample material. Each micro sensor contains two excitation coils, two sensing coils, and leads to connect the coils electrically to contact pads at the backside of the sensor. Two key requirements have to be fulfilled to achieve a sensor sensitivity as high as possible. First, the magnetizing field generated in the measuring gap of the sensor must be as homogeneous as possible. Second, the applied soft-magnetic material must have a high relative permeability $\mu_{r}$ to reach a high sensitivity during the detecting of the $B-H$ loop $^{4}$.

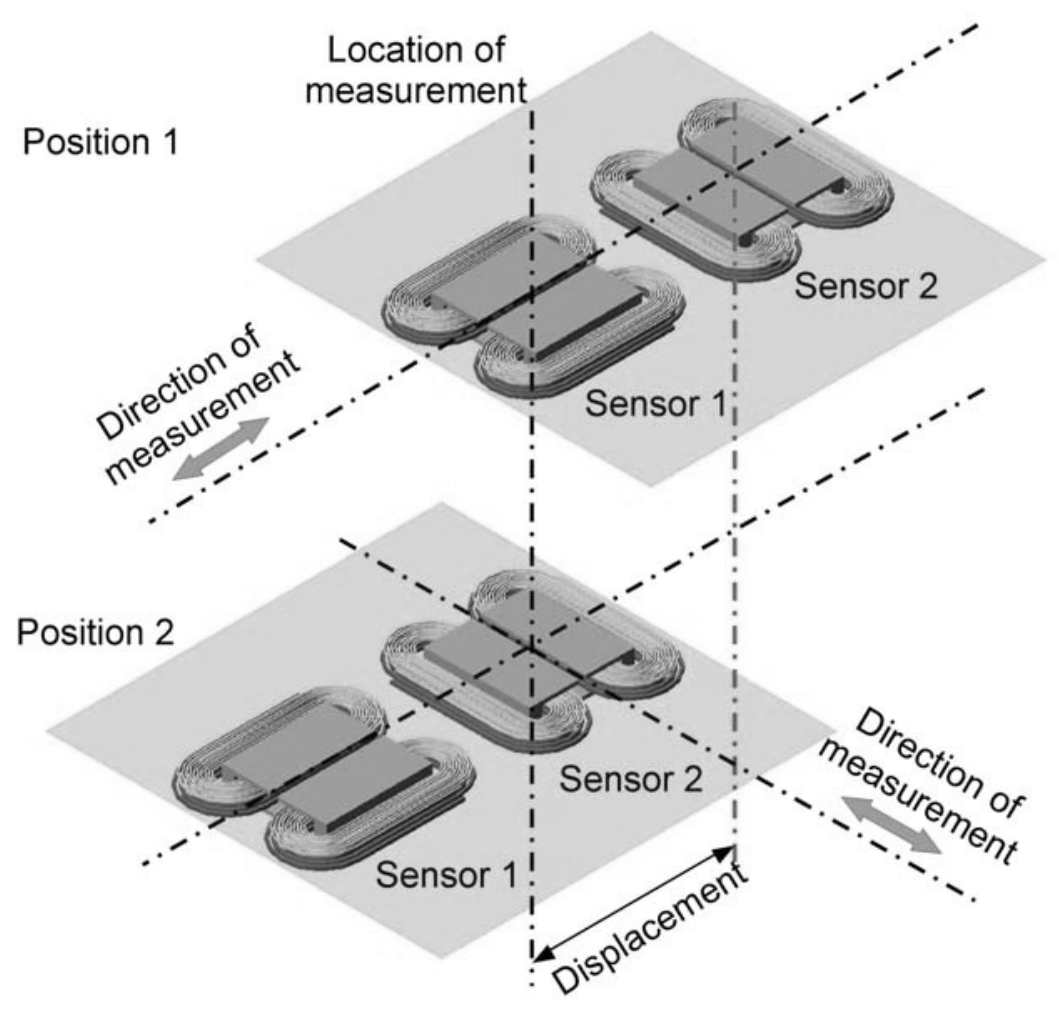

Fig. 1: Arrangement of a pair of micro hysteresis sensors capable of measuring in two directions offset by $90^{\circ}$ at the same location

\section{Modular Magnetic Micro Sensor Family}

A family of magnetic sensors covering different measuring capabilities was created by using standardized, modular components. These sensors are fabricated in thin-film technology with a standard set of process steps. To demonstrate this approach, a magnetic sensor family with members capable of

\footnotetext{
${ }^{3}$ D. Dinulovic, E. Flick, H. Gerdes, K. Feindt, M. Eccarius, H.H. Gatzen: Concept for a Wafer Level Test System for Measuring Magnetic Film Properties. Symposium on Magnetic Materials, Processes and Devices. $210^{\text {th }}$ Meet. of The Electrochemical Society, Cancun, Mexico, 2006, ecs transactions, Vol. 3, Issue 25, pp. 165-177, 2007

${ }^{4}$ E. Flick, K. Feindt, H.H. Gatzen: Micro Hysteresis Sensor for Wafer Level Testing of Magnetic Films. Intermag, Conf. 2008, Madrid, Spain, BC-04, 2008; accepted for publication in IEEE Trans. on Magn.
} 
serving as a strain gauge sensor for strain or force measurements as well as a sensor for analyzing magnetic material properties is presented ${ }^{5}$. In principle, the three sensors follow the same design and only differ regarding the integration of the magneto-elastic components. The respective sensor is composed of modular components enabling to build the respective micro sensors. The main advantage of the modular sensor concept is that alternative micro sensors intended for different sensing applications can be fabricated within one fabrication run on the same wafer because the fabrication sequence only differs in the last process step where the magneto-elastic element is fabricated.

Figure 2 presents the schematics of the modular micro sensor familiy belonging to this strain gauge sensor for direct strain measurement (the sensor is integrated into the strained component - Fig. 2a), a remote strain gauge sensor where only the magneto-elastic element is subjected to strain (Fig. 2b), and an eddy current sensor with the same basic sensor design but no magneto-elastic element at all (Fig. 2c). All the sensors feature an E-shaped core consisting of a lower flux guide and three poles guiding the magnetic flux either into the magneto-elastic sensing film or the probe material.

a)

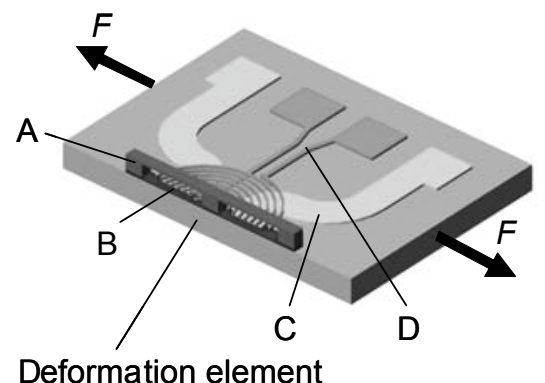

c)

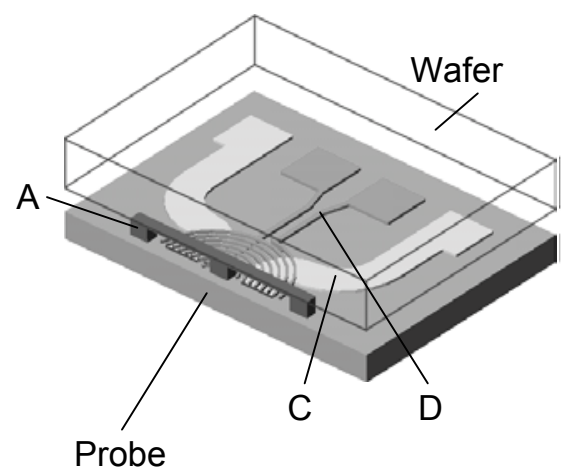

b)

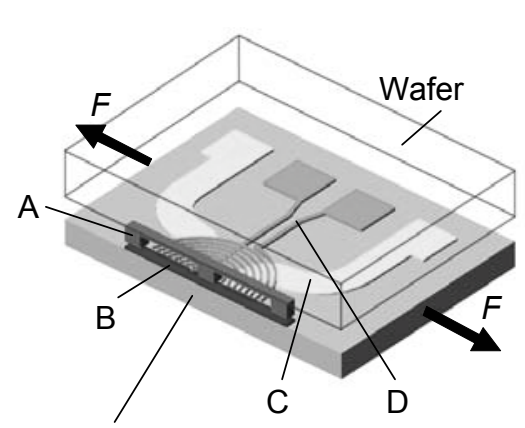

Deformation element

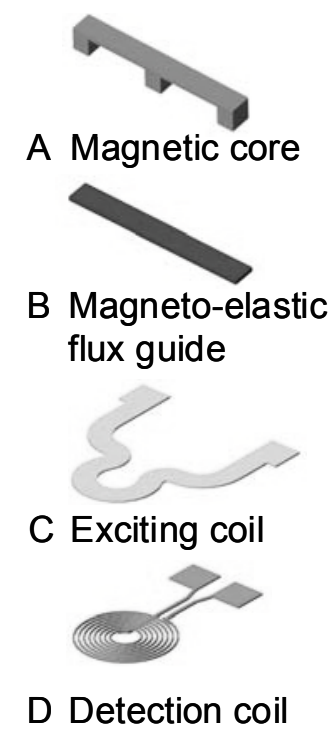

Fig. 2: Modular magnetic sensor: a) Direct strain gauge sensor; b) Remote strain gauge sensor; c) Eddy current sensor

The sensing principle is based on the VILLARI effect (also called magneto-elastic effect) which is the inverse effect to JOULE`s magnetostriction. Exposing a sample made of a magnetic material to strain

\footnotetext{
${ }^{5}$ S. Hansen, L. Rissing, H.H. Gatzen: A Concept for a Toolbox of Components for Modular Magnetic Microsensors. Symposium on Magnetic Materials, Processes and Devices, $210^{\text {th }}$ Meet. of The Electrochemical Society, Cancun, Mexico, 2006, ecs transactions, Vol. 3, Issue 25, pp. 235-246, 2007
} 
causes a change of the magnetic properties of the sample ${ }^{6}$. In particular, the magnetic permeability $\mu_{r}$ is affected by the change of the magnetic properties. This phenomenon is used in the micro transformer serving as an inductive strain gauge similar to the one described to measure a mechanical force, pressure, as well as strain ${ }^{7}$. For such a device, one leg of the micro transformer consists of the magnetoelastic alloy NiFe45/55 featuring a positive coefficient of magnetostriction. $\lambda$ strongly depends on the composition of the alloy and is at a maximum for NiFe45/55. Figure 3 depicts a micrograph of the micro transformer used as a strain gauge. A single turn excitation coil with a large cross section induces an alternating flux, while a multi-turn detection coil picks up the voltage induced by the periodic flux change, which ultimately is the output signal.

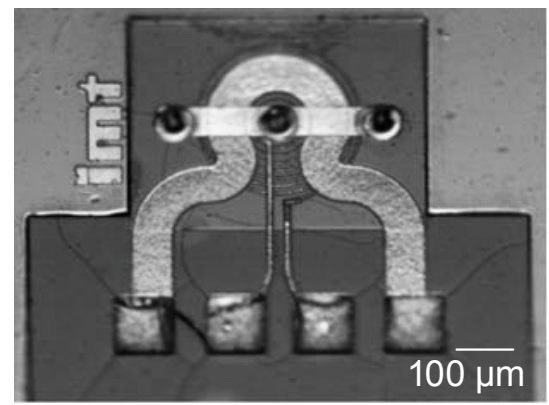

Fig. 3: Micrograph of a micro strain gauge

\section{Carbon Fiber Strain Gauge}

An approach towards nanotechnology is integrating a strain gauge into a carbon fiber, allowing to measure the strain in composite materials. Such an approach represents an alternative to applying planar technologies for fabricating a strain gauge ${ }^{9,10}$. High strength carbon fibers are used as reinforcement for advanced composite low-weight materials to be used in space and the aircraft industry, for example. The mechanical stress in the fibers are part of the key data to be known for an optimal design of composite material components.

To measure the strain in carbon reinforced materials, a carbon fiber is covered by electroplating with a thin-film made of magneto-elastic NiFe45/55. On top of the NiFe45/55 layer, an inorganic $\mathrm{Si}_{3} \mathrm{~N}_{4}$ layer is deposited by PECVD serving as an insulation followed by a Cu layer fabricated by magnetron sputtering and patterned to create a pair of coils. The coils' task is to detect the fiber's state of strain. For the fabrication of the coil structure, nanofabrication techniques are required. The proposed tool consists of an ultra precision fiber turning lathe in combination with a Focused lon Beam (FIB) system. The lathe turns the carbon fiber, while the focused ion beam serves as a nanocutting tool, cutting a spiral into the $\mathrm{Cu}$, thus creating the coil. Due to the small fiber diameter of $8 \mu \mathrm{m}$, handling is very challenging. A docking station allows to operating the lathe outside the FIB system. With the lathe docked to this station, carbon fiber mounting and other preparation steps outside of the FIB can be performed ${ }^{10}$. Figure 4 schematically shows a cross section of a carbon fiber bundle covered with a magnetostrictive NiFe45/55 layer followed by an the $\mathrm{Si}_{3} \mathrm{~N}_{4}$ insulation layer and a Cu layer which was patterned using FIB technology to create the coils.

\footnotetext{
${ }^{6}$ A. Ben Amor, C. Ruffert, H.H. Gatzen: NiFe 45/55 and its Application in a Strain Gauge Sensor. Proc. $8^{\text {th }}$ Int. Symposium on Magnetic Materials, Processes and Devices, $206^{\text {th }}$ Meet. of The Electrochemical Society 2004, Honolulu, Hawaii, USA, pp. 481-492, 2004

${ }^{7}$ T. Griesbach, L. Rissing, H. H. Gatzen: Integration of a Temperature Sensor into a Modular, Multifunctional Micro Sensor Family. I PROMS 2008, the $4^{\text {th }}$ Virtual Conference, of the EU-funded FP6 I PROMS Network of Excellence on Innovative Production Machines and Systems, 1 and 14 July 2008, (accepted)

${ }^{8}$ E. Kneller: Ferromagnetismus. Springer Verlag, Berlin, 1962

9 D. Dinulovic, H. Gerdes, H. Mucha, B. Wielage, H.H. Gatzen: Carbon Fiber with Magnetoelastic Sensing Capability for Composite Materials. Sensor Letters, Vol. 5, No. 1, pp. 218-221, 2007

${ }^{10}$ A. Belski, W. Kammrath, H.H. Gatzen: Focused Ion Beam Fiber Turning Lathe for Fabricating a Strain Gauge for Carbon Fibers. Proc. EUSPEN $8^{\text {th }}$ Int. Conf. 2008, Zurich, Switzerland, Vol. 2, pp. 129-133, 2008
} 
a)

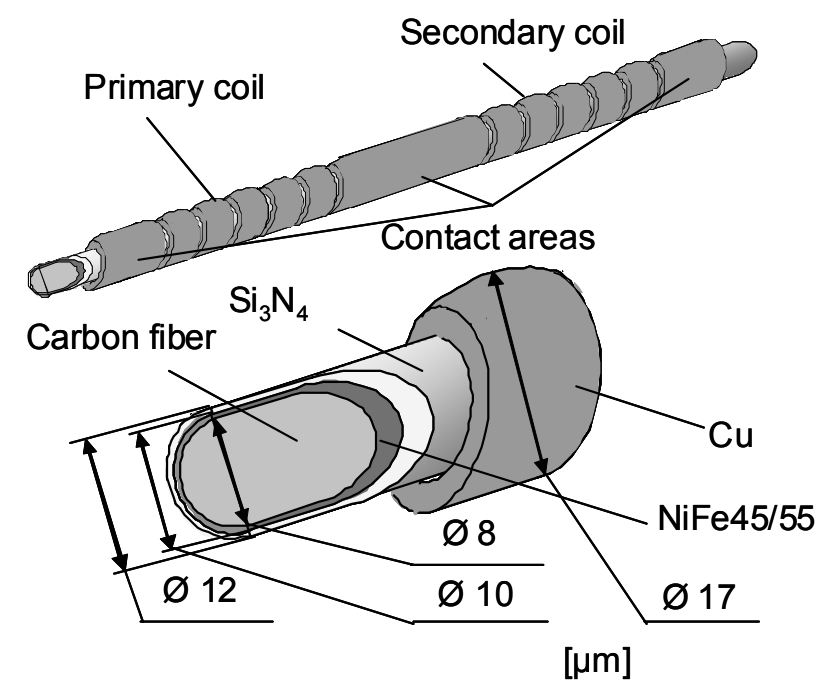

Fig. 4: Schematic representation of the carbon fiber micro strain sensor a) view; b) cross section

Figure 5 shows an SEM micrograph of the three-layered carbon fiber strain gauge on the surface of the carbon fiber. The thin-films are deposited applying electroplating for the magneto-elastic NiFe45/55, PECVD for the inorganic $\mathrm{Si}_{3} \mathrm{~N}_{4}$ insulation, and electroplating for the coil material $\mathrm{Cu}^{11}$.

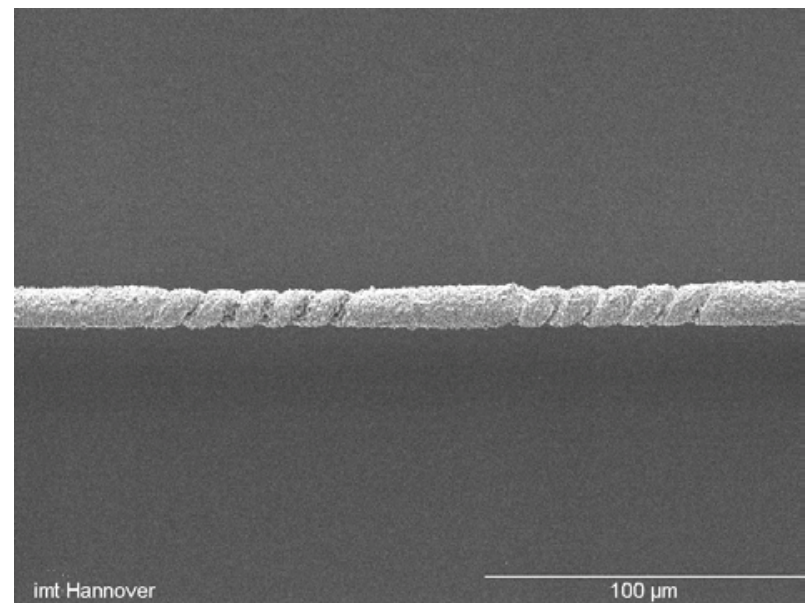

Fig. 5: Prototype of a carbon fiber strain gauge

\section{Micro Inductosyn ${ }^{\mathrm{TM}}$ Position Measurement System}

Inductive measuring systems fabricated in thin-film technology feature a large degree of integration, miniaturization, and low manufacturing costs. An inductive micro position measuring system based on the Inductosyn ${ }^{\mathrm{TM}}$ principle was developed and fabricated ${ }^{12}$. The sensor consists of three coil systems. The magnetic scale is positioned on the stationary part of the system, the stator, and is facilitated using a planar meander coil. It contains of two planar meander coils which are located opposite and parallel to the coil system of the scale. If the excitation coil is located congruently with the sensing coil, an optimal coupling between the coils results in a maximum of the induced voltage. To increase the coupling between the excitation and sensing coils of the micro device, a soft magnetic flux guide has been

\footnotetext{
${ }^{11}$ H.H. Gatzen: Magnetic Micro Electro-mechanical Systems for Sensor and Actuator Applications. PRiME 2008 Meeting, Honolulu, HI, USA, The Electrochemical Society, MA 2008-2, Abs. 2601.pdf, 2008 12 D. Dinulovic, D. Herrmann, J. Flügge, H.H. Gatzen: Development of a Linear Micro Inductosyn Sensor. IEEE Trans. on Magn., Vol. 42, No. 10, pp. 2830-2832, 2006
} 
integrated. Such a flux guide is not present on the macroscopic Inductosyn ${ }^{\mathrm{TM}}$ system. The sensing part of the system is positioned on a movable part, the traveler. The system output are two signals which are offset by $\pi / 2$ to allow for a detection of the direction of motion. Figure 6 depicts a schematic representation of the micro Inductosyn ${ }^{\mathrm{TM}}$ system. Figure 6a principally shows a cross section of the exciting coils in the stator arranged opposite the sensing coils in the traveler. Figure $6 \mathrm{~b}$ displays the two output signals (sine and cosine) offset by $\pi / 2$ as a function of the displacement of the traveler relative to the stator.

a)

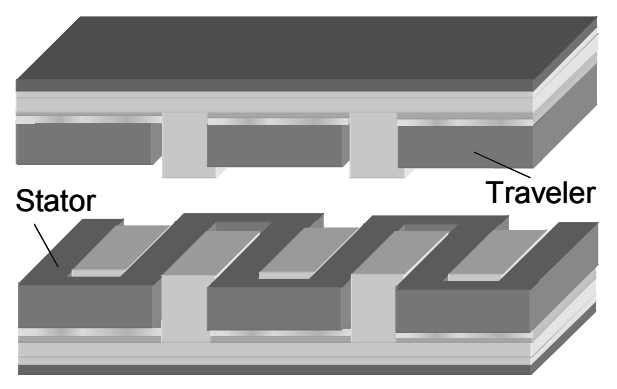

b)

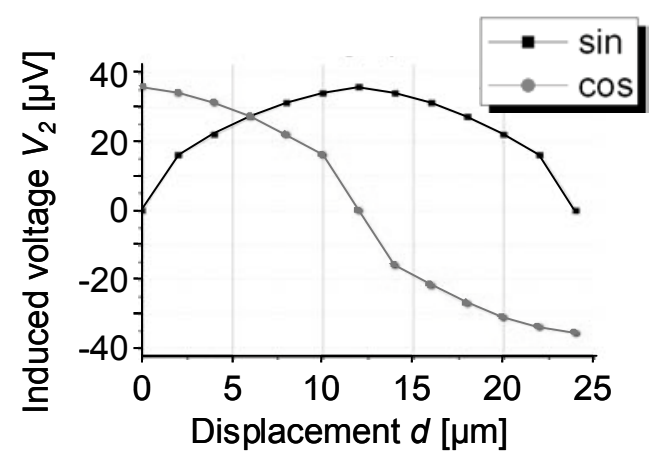

Fig. 6: Principle of the micro Inductosyn ${ }^{\mathrm{TM}}$ system: a) Coil system and b) output signal as a function of the displacement of the traveler ${ }^{12}$

The dimensions of the traveler amount to $0.6 \mathrm{~mm} \times 4.5 \mathrm{~mm}$ and to $1 \mathrm{~mm} \times 3 \mathrm{~mm}$ for the stator. The travel range is $4 \mathrm{~mm}$ and the resolution $2 \mathrm{~nm}$. The micro position measurement system can thus detect the relative movement of machine components such as the position of a pneumatic valve with an extremely high precision.

\section{Conclusion}

This paper demonstrates the versabiliy of magnetic devices for test purposes. For the determination of thin-film magnetic properties on wafer level, a measurement system for determining could be devised allowing to determine the magnetic properties along the easy and the hard axis at a test location. A concept for a modular sensor approach is presented based on a micro strain gauge sensor design applicable for direct and remote strain measurements as well as the detection of eddy currents. The modular design is a promising concept to simplify the design and fabrication process of the devices. The research executed so far in the area of magneto-elastic functional coatings demonstrate, that the FIB preparation of a carbon fiber strain gauge is a promising approach for instrumenting carbon fibers. Combining an ultra precision lathe with FIB machining provides a novel method for machining non-planar components. An inductive position measurement system demonstrates the potential for the miniaturization of the Inductosyn ${ }^{\mathrm{TM}}$ principle by scaling down the geometry and integrating a softmagnetic flux guide.

\section{Acknowledgment}

This research was sponsored in part by the German Federal Ministry of Education, Research, and Technology (BMBF) within the framework of the program "MST Pruef" (project CHARMA), the DFG (German Research Foundation) within the Collaborative Research Center (SFB) 653 "Gentelligent Components in Their Lifecycle - Utilization of Inheritable Component Information in Product Engineering", the DFG within the project "Magnetoelastic Sensors for Health Monitoring of Composite Materials by Mechanical Stress", and the DFG within the project "Research and Development of a High-Resolution Inductive Measurement System" (Micro-Inductosyn ${ }^{\mathrm{TM}}{ }^{\text {). }}$. 